\title{
De quoi Intégration est-il le nom ? L'importation d'une querelle de mots dans le champ de la formation linguistique des migrants
}

What Does 'Integration' Stand For? The Introduction of a Verbal Dispute in the Field of Instructional Activity for the Benefit of Adult Migrants

\section{Maude Vadot}

\section{(2) OpenEdition Journals}

\section{Édition électronique}

URL : http://journals.openedition.org/aad/2228

DOI : 10.4000/aad. 2228

ISSN : 1565-8961

\section{Éditeur}

Université de Tel-Aviv

\section{Référence électronique}

Maude Vadot, « De quoi Intégration est-il le nom ? L'importation d'une querelle de mots dans le champ de la formation linguistique des migrants ", Argumentation et Analyse du Discours [En ligne], 17 | 2016, mis en ligne le 15 octobre 2016, consulté le 23 septembre 2019. URL : http://journals.openedition.org/ aad/2228; DOI : 10.4000/aad.2228

Ce document a été généré automatiquement le 23 septembre 2019.

\section{(c) (i) $\odot$}

Argumentation \& analyse du discours est mis à disposition selon les termes de la licence Creative Commons Attribution - Pas d'Utilisation Commerciale - Pas de Modification 4.0 International. 


\title{
De quoi Intégration est-il le nom? L'importation d'une querelle de mots dans le champ de la formation linguistique des migrants
}

\author{
What Does 'Integration' Stand For? The Introduction of a Verbal Dispute in the \\ Field of Instructional Activity for the Benefit of Adult Migrants
}

Maude Vadot

La thématique de l'immigration est en France un lieu particulier, objet privilégié de l'agenda politico-médiatique comme des discours ordinaires. Lieu d'affrontements idéologiques en constante reconfiguration, elle est également le théâtre de querelles de mots récurrentes. Si certaines font irruption à la faveur d'événements particuliers, à l'instar des contestations de la formule Identité nationale en 2009-2010 ou de l'opposition Migrant / Réfugié dans l'été 2015, d'autres sont plus anciennes : il en est ainsi des débats portant sur l'emploi des termes du paradigme Insertion, Intégration, Assimilation, auquel on adjoint parfois Inclusion ou Acculturation.

Cette querelle de mots a fait irruption dans le champ de la formation linguistique des migrants adultes à la faveur de son institutionnalisation progressive. Les dernières étapes en date de ce processus d'institutionnalisation ont en effet consacré l'usage du terme Intégration en l'employant de manière récurrente, et ce malgré les remaniements gouvernementaux dont auraient pu découler des évolutions terminologiques. Ainsi, c'est par décision du Ministère de l'Immigration, de l'Intégration, de l'Identité nationale et du Développement solidaire, et dans le cadre de la généralisation du Contrat d'Accueil et d'Intégration le 1er janvier 2007 que le suivi d'un module linguistique est devenu obligatoire pour une partie des migrants extra-européens. Sa mise en œuvre a été confiée à l'office Français de l'Immigration et de l'Intégration (OFII), créé en 2009. En 2011, c'est en réponse à une commande de la Direction de l'Accueil, de l'Intégration et de la Citoyenneté (DAIC) qu'a été publié le Référentiel FLI - 
Français Langue d'Intégration (Vicher et al. 2011), ayant pour visée annoncée l'amélioration de la qualité des formations dispensées aux adultes migrants.

Le référentiel et les annonces qui l'ont accompagné ont alors fait l'objet de contestations de la part d'une partie du monde associatif et universitaire (Huver et al. 2011, «Flic ? " 2011, Fédération des centres sociaux de Paris 2012, Bruneau et al.2012), sans que cela n'aboutisse toutefois à l'annulation des décrets. Les critiques formulées ont concerné des aspects didactiques de la démarche exposée, mais ont également dénoncé l'ingérence du Ministère de l'Intérieur dans les formations universitaires ainsi que l'idéologie assimilationniste décelable dans le texte, notamment à travers la place centrale donnée à la langue et l'accent mis sur l'enseignement des valeurs de la République.

A la suite de Siblot (2001a : 195), on désignera par « nomination » l'acte discursif réalisé par l'énonciateur', et par «dénomination" son produit en langue, porteur d'un programme de sens capitalisé au travers de nominations répétées. Dans le cas étudié ici, on a donc affaire à une double nomination : en publiant le référentiel, le Ministère en charge du projet réalise un premier acte discursif créant la désignation Français Langue d'Intégration, relativement stabilisée depuis. Mais il actualise par là une dénomination particulière, celle d'Intégration: il s'agit là d'un second acte que l'on étudiera en tant que tel.

Le présent article vise à étudier l'articulation entre argumentation et nomination à partir du conflit nominatif réactivé par la parution du référentiel, dans une perspective d'analyse $\mathrm{du}$ discours praxématique et sociolinguistique. Après avoir resitué brièvement la querelle opérant au sein du paradigme désignationnel auquel Intégration appartient (partie 1), les analyses poursuivront un double objectif. Il s'agira d'une part de décrire une situation dans laquelle le mot n'est pas un instrument au service de l'argumentation, mais en devient un enjeu (Micheli 2013) : par quelles stratégies sociodiscursives les locuteurs légitiment-ils et justifient-ils leur choix (parties 2 et 3) ? On cherchera d'autre part à éclairer le conflit en mettant au jour, dans une démarche sémasiologique inspirée de l'analyse du discours à entrée lexicale, des éléments du programme de sens du praxème Intégration que les auteurs, de façon dialectique, actualisent et contribuent à la fois à imposer dans le champ de la formation linguistique (partie 4).

On exclura des analyses l'avant-propos du référentiel (p. 4-5), dont les auteurs ${ }^{2}$, identifiés par une signature individuelle, n'appartiennent pas au collectif d'experts. Ce dernier s'étant désolidarisé des propos tenus («Français langue d'intégration (FLI) », 2011), cette restriction du corpus correspond à la volonté d'analyser uniquement ce dont le collectif d'experts accepte la responsabilité.

\section{Des querelles de mots anciennes}

Le conflit de nomination qui nous préoccupe n'est pas nouveau, et de nombreux auteurs ont cherché à le documenter. On se bornera ici à indiquer quelques éléments qui entrent en résonance avec l'objet du présent article, qui signalent «le poids d'autres discours qui se sont en quelque sorte "empilés" " (Moirand 2003 : 45) dans les dénominations considérées.

Une première caractéristique de la nomination Intégration est qu'elle est mobilisée dans différents types de discours. On doit ainsi différencier ses usages en sociologie, 
discipline qui prétend caractériser et objectiver le réel, de ses usages en politique, qui visent à définir des objectifs et des obligations, donc possède un caractère plus directement normatif. Par conséquent, "on ne sait jamais très bien, dès lors, si [l'intégration] constitue une valeur, un enjeu qu'il faudrait atteindre, un horizon, ou une caractéristique objective d'un modèle réel » (Wieviorka $2001: 20$ ).

Par ailleurs, de nombreux auteurs soulignent la fluctuation des termes en usage. Il est ainsi possible de dégager des périodes d'emploi préférentiel de chaque nomination dans la presse (Bonnafous 1992), les débats parlementaires (Gaspard 1992) ou l'action publique (Logez 2005), où les changements de paradigme ont des conséquences en matière de travail social (Lemière 2006). Les termes ont également circulé d'un camp politique à un autre, révélant des évolutions dans les positions idéologiques mais également dans les contenus sémantiques (Gaspard op. cit.). Bonnafous (op. cit.) identifie ainsi une confusion terminologique et conceptuelle, pendant que Laacher et Sayad (1999) considèrent que les usages révèlent que les termes sont à la fois interchangeables et rapidement renouvelés. Les mêmes auteurs s'attachent pourtant à les différencier du point de vue du contenu idéologique dont ils sont originellement porteurs, rejoignant ainsi Lapeyronnie (2003) et Lochak (2006). Pour le premier, Intégration exprime le point de vue du dominant sur le dominé, tandis que pour la seconde, les termes Insertion, Intégration et Assimilation vont du plus respectueux au plus impérialiste. Par ailleurs, si les politiques d'action sociale instaurées à partir des années 70, désignant par Insertion des programmes en faveur de l'emploi, donc de l'insertion dite professionnelle, peuvent influer sur les emplois actuels, la problématique a très rapidement été mise en lien avec celle de l'insertion dite sociale, et progressivement assimilée à la notion d'intégration des individus dans la société, ce phénomène contribuant lui aussi au brouillage sémantique (Dubar 1999).

Ainsi, bien que l'étymologie et l'histoire de l'apparition de ces trois termes révèlent des conceptions différentes des rapports entre l'étranger et la communauté d'accueil (Grange 2005), dans la pratique des usages, Insertion et Intégration semblent désormais souvent nommer la même réalité, voire être interchangeables. Pour autant, l'exploration du sentiment linguistique de certains locuteurs ${ }^{3}$ permet de faire apparaître le poids de mémoires discursives différenciées se rapportant à chacune des dénominations mises en contraste (Vadot, à paraitre). Si la querelle fait couler beaucoup d'encre, c'est qu'elle informe de multiples domaines sociaux : vie politique et médiatique, action sociale et politiques publiques, modélisation sociologique, didactique et politique de formation linguistique (Castellotti 2008). En cela, elle illustre bien le fait que les nominations peuvent constituer des enjeux brûlants.

\section{Français Langue d'Intégration : une nomination juridique}

Comme l'ont souligné les critiques évoquées plus haut, la parution du référentiel met en saillance les rapports de pouvoir au sein du champ de la formation linguistique des migrants. Dans un domaine qui a d'abord reposé sur des initiatives de la société civile (Leclercq 2012) et dans lequel les contraintes qui s'exercent ont des effets délétères sur les contenus de formation (Étienne 2004 ; Vadot 2011, Lebreton 2014) et sur les conditions de travail des formateurs (Vadot 2014), identifier ceux qui définissent les orientations d'une politique de formation constitue un enjeu important. 
De la même façon, la nomination FLI pose la question du processus à l'œuvre et de l'identité des acteurs-'nommeurs' : qui attribue les noms, en vertu de quoi, et avec quels effets? Ces questions sont d'autant plus saillantes qu'il s'agit d'une renomination : le champ désormais désigné par le sigle FLI existait avant la création de ce dernier, ce sur quoi les auteurs du référentiel s'appuient pour justifier leur acte. Pour eux, la nomination qu'ils effectuent est rendue nécessaire par le degré de maturité et d'autonomie atteint par le champ, et vise à le discrétiser :

Le terme FLI donne un nom à une réalité qui s'est construite et autonomisée par rapport aux autres champs didactiques et professionnels qui lui sont proches. Le champ professionnel de la formation linguistique d'adultes migrants s'est spécifié ces dernières années. Il est désormais nécessaire de l'identifier et d'en délimiter les contours de façon précise (Vicher et al. $2011: 9)^{4}$.

On peut rapprocher cet acte discursif de la re-nomination réalisée en 2009 au moyen du décret $n^{\circ}$ 2009-331 «substituant la dénomination "Office Français de l'Immigration et de l'Intégration" à la dénomination "Agence Nationale de l'Accueil des Étrangers et des Migrations" ». Cet intitulé transparent montre que l'opération de création de l'OFII était en réalité un acte de re-nomination de ce qui se nommait alors ANAEM, dont les fonctions ont été parallèlement restructurées (loi $n^{\circ}$ 2009-323 parue le même jour). Il est par conséquent tentant de s'interroger sur la pertinence véritable de la renomination en Français Langue d'Intégration d'un domaine qui préexistait à ce baptême.

\subsection{Une situation d'énonciation dans laquelle entre en jeu le pouvoir de l'institution}

Contrairement à un ouvrage pédagogique classique, le référentiel FLI est un texte institué, un discours de cadrage qui tire son autorité de la commande institutionnelle à laquelle il répond et dont il se revendique : l'avant-propos est signé de deux hauts responsables institutionnels et le logo de l'institution commanditaire apparait sur la dernière page. Les auteurs, bien qu'identifiés nommément, forment un collectif d'énonciation légitimé par l'institution, qui les place en position d'«experts " du domaine (p. 2) : elle leur apporte donc sa caution tout autant qu'ils lui font bénéficier de leur légitimité scientifique, les uns et les autres construisant ainsi par leur influence réciproque une posture d'autorité solidement assise. Forts de cet ethos préalable (Amossy 2006: 79), les auteurs mettent par ailleurs en avant la continuité institutionnelle et scientifique dans laquelle leur travail s'inscrit, tout en évitant soigneusement d'identifier les acteurs des procès qu'ils rapportent (p. 7), ce qui leur permet d'apparaître comme les seuls experts qualifiés du domaine, alimentant ainsi par des procédés discursifs cet ethos préalablement déclaré. Enfin, la parution du document s'est appuyée sur un dispositif légal reposant sur plusieurs textes parus au journal officiel : le référentiel lui-même a été défini par l'arrêté du 25 novembre 2011 ; il avait été précédé, quelques semaines auparavant, par le décret $n^{\circ}$ 2011-1266 relatif à la création du label FLI - délivré par l'État sur la base du référentiel éponyme. Comme tout label, ce dernier apparaît comme gage d'une expertise, qui ne peut être délivré que par des personnes elles-mêmes expertes.

A travers cet arsenal juridique, l'institution s'est donc dotée des moyens nécessaires pour que la nomination $F L I$ ait des implications tangibles dans la réalité extralinguistique : $d u$ fait du renforcement de ses effets pragmatiques par un cadre juridique contraignant, elle constitue tout autant un acte de baptême institutionnel 
qu'un discours agissant, discrétisant un champ et des objets, et impliquant des conséquences pour les acteurs du domaine ainsi que les publics bénéficiaires. Le sigle créé visait ainsi à dénommer tout autant un champ professionnel et une politique linguistique (p. 9), qu'un objet (« une langue horizon», p. 10 ; «le français, langue d'intégration sociale, économique et citoyenne ", p. 11), une "démarche didactique " (p. 13), des métiers spécifiques ( formateur FLI» et " coordinateur FLI», p. 13) et un label (p.17). Le label ayant été présenté comme une condition d'obtention des futurs marchés publics ${ }^{5}$, de très nombreux organismes ${ }^{6}$ se sont engagés à leurs frais dans une démarche de labellisation; de même, des formations FLI destinées aux formateurs ont été organisées partout en France, parallèlement à la création de diplômes universitaires ou de masters mention $\mathrm{FLI}^{7}$. Des entretiens menés auprès de formateurs en 2013 révèlent qu'une partie d'entre eux se présentait alors comme «formateur FLI »; dans les offres diffusées par Pôle Emploi, les organismes ont recours à cette dénomination. Enfin, le décret instituant le référentiel FLI est paru conjointement au décret $n^{\circ}$ 2011-1265 qui fixe à B1 le niveau de français nécessaire pour être naturalisé: le référentiel y fait d'ailleurs référence (p. 10). L'ensemble de ces faits montre à quel point cette nomination, qui repose sur un pouvoir institutionnel structuré et doté de moyens juridiques spécifiques, est intimement liée à un ensemble de domaines allant de la politique d'accueil et d'accompagnement des étrangers à la politique de qualification des salariés (formation initiale et continue, universitaire ou non), en passant par le code de la nationalité.

\subsection{Nomination et prescription}

La nomination FLI réalise l'affirmation du choix d'un terme du paradigme présenté supra: la dénomination Français Langue d'Intégration, destinée à remplacer les précédentes - par exemple celles d'Alphabétisation ou de Cours de FLE/FLS pour migrants vient ainsi confirmer la validité, aux yeux de l'État, de la dénomination Intégration. Mais les auteurs et les institutions pour lesquelles ils agissent ne se contentent pas d'employer ce mot : ils l'imposent au moyen de l'arsenal juridique décrit plus haut, ce qui constitue une étape supplémentaire dans le processus de prescription de la dénomination par le pouvoir institué. Cette nomination est donc une manifestation de la violence symbolique exercée par l'État afin de se maintenir, et qui s'exprime à travers la production et l'imposition de catégories qui semblent ensuite naturelles aux locuteurs alors qu'elles sont culturellement arbitraires (Bourdieu 1993):

A travers l'encadrement qu'il impose aux pratiques, l'État instaure et inculque des formes et des catégories de pensée communes, des cadres sociaux de la perception, de l'entendement ou de la mémoire, des structures mentales, des formes étatiques de classification. Par là, il crée les conditions d'une sorte d'orchestration immédiate des habitus qui est elle-même le fondement d'une sorte de consensus sur cet ensemble d'évidences partagées qui sont constitutives du sens commun (ibid. : 59).

Cette violence symbolique repose ici sur la conjonction du capital symbolique (existence d'une institution nationale spécialisée et recours à des experts nommés par cette dernière) et du capital juridique de l'État. Si elle constitue une étape dans la querelle durable pour l'imposition d'un des termes du paradigme, la bataille ne se joue pas à armes égales : les auteurs et l'institution s'appuient sur un agencement structurel de pouvoir qui leur permet d'imposer le double acte de nomination qu'ils réalisent, et d'en faire le vecteur d'une reconfiguration symbolique et pratique du champ. 
Cependant, les auteurs ne se contentent pas d'user du pouvoir de l'institution pour légitimer leur choix : conscients de la polémique dans laquelle ils se positionnent, ils prennent également en charge de manière dialogique la justification de ce qu'ils font.

\section{La nomination Intégration : un choix justifié par les auteurs}

La praxématique analyse l'opération de nomination comme réalisant deux actes discursifs simultanés. Le premier consiste à prédiquer l'existence de l'objet, à le poser comme existant en le nommant ${ }^{8}$. Le second consiste à poser que ce qu'on nomme s'appelle ainsi qu'on le nomme, en opérant un choix parmi un paradigme désignationnel. En cela, le locuteur nomme, de façon contrastive, son rapport à l'objet (Siblot 2001a).

Toute nomination définit de plus un positionnement du locuteur par rapport aux autres locuteurs qui nomment, ont nommé ou nommeront l'objet en question. En actualisant une dénomination, le locuteur réactive des éléments de points de vue antérieurs, et s'ajuste à l'interlocuteur en anticipant parfois sur des éléments de points de vue à venir; ce que la praxématique analyse comme la manifestation d'un dialogisme de la nomination (Siblot 2001b). L'analyse de la nomination comme acte discursif et expression d'un point de vue a conduit Siblot (2007) à considérer une deixis propre à l'acte de nommer et à la catégorisation nominale, afin de souligner le positionnement subjectif du locuteur manifesté par l'acte de nomination.

Cette modélisation est d'autant plus appropriée dans le cas d'une nomination conflictuelle, situation qui amène les locuteurs à expliciter et à défendre leur positionnement en l'argumentant, et dans laquelle les mots doivent être considérés comme l'enjeu même de l'argumentation (Micheli 2013). En l'occurrence les auteursnommeurs, conscients de la mémoire discursive de la dénomination choisie, savaient que le terme employé était contestable : ils écrivent, sous le sous-titre «Pourquoi une langue d'intégration?», que "l'utilisation du terme "intégration" peut susciter un débat » (p. 8). Dans cette section d'une trentaine de lignes, les auteurs argumentent en faveur du terme choisi en anticipant les réfutations de leurs lecteurs, ce en quoi se manifeste la dimension interlocutive de la nomination (Chanay 2001: 178). On distinguera trois aspects du processus de justification : la neutralisation des opposants, l'appel au pragmatisme et l'éviction silencieuse des autres termes.

\subsection{Surénonciation et neutralisation des opposants}

Évoquant l'histoire de la formation linguistique des migrants, les auteurs ne nomment aucun acteur, ce qui leur permet d'effacer toute conflictualité et d'apparaître comme seule volonté agissante (voir plus haut 2.1). Il n'en va pas de même dans la section analysée ici, au sein de laquelle les auteurs font intervenir deux groupes de locuteurs génériques, donnant à l'argumentation déployée une visée explicite de positionnement (Micheli 2012) :

le terme «intégration » [...] renvoie à l'existence de deux pôles antagonistes : celui du relativisme culturel radical et celui du modèle de société dit "républicain", qui prévaut en France (Vicher et al., p. 8). 
Les auteurs sont alors en position de surénonciateur (Rabatel 2003), position dominante qui permet au locuteur citant de rapporter à son avantage des propos et des points de vue en leur imprimant sa marque. Bien qu'ils maintiennent le cap d'une énonciation désembrayée et donc d'un effacement énonciatif le plus efficace possible, le point de vue des auteurs est palpable à travers leur présentation des " pôles » qui s'affrontent. En effet, si le second est présenté comme un "modèle de société » qui "prévaut en France», donc ancré dans une situation matérielle géographiquement située, le premier ne bénéficie pas de cet ancrage : il est donc relégué au rang d'idéologie et qualifié de "radical», adjectif pouvant marquer la péjoration. La phrase suivante conserve cette orientation axiologique en opposant «valeurs humaines fondamentales» et "différences culturelles ou communautaires». Alors que le "modèle républicain " est en position de sujet syntaxique, le "relativisme culturel ", qui n'est plus nommé comme tel, devient le terme d'une comparaison, ce qui revient à présenter le premier en plein et le second en creux :

Ce dernier met l'accent, non sur les différences culturelles ou communautaires, mais sur l'universalité de valeurs humaines fondamentales et de l'individu qui est

d'abord un citoyen avant d'être membre d'un groupe culturel particulier (p. 8).

Les auteurs continuent en présentant les «tenants d'un modèle de société multiculturelle et différentialiste " comme "réticents à toute forme d'assimilation, voire d'acculturation ou même d'intégration des groupes particuliers " et en les opposant aux «tenants du modèle républicain qui voient, dans les différences culturelles revendiquées, un risque de communautarisation de la société et d'éclatement de la collectivité des citoyens " (p. 8). Les premiers sont donc associés à une attitude négative susceptible d'aller à l'encontre de la volonté même des "groupes particuliers » dont il est question, tandis que les seconds sont caractérisés par le souci du bien commun et de la conservation du collectif - dont l'existence n'est pas discutée.

Si par l'ensemble de ces procédés, les auteurs semblent se positionner résolument contre l'un des deux pôles concernés et, en miroir, en faveur du second, ils concluent cette présentation partiale en se plaçant au-dessus de la mêlée, ce qui leur permet, comme on va le voir, de valider de fait la nomination Intégration.

\subsection{Emplois standard ou autonymique et appel au pragmatisme}

Le passage étudié ici mêle deux emplois distincts de la dénomination Intégration: si le premier paragraphe (cité plus haut) s'ouvre sur un emploi autonymique marqué par le recours aux guillemets, les deux suivants font apparaître trois occurrences en emploi standard. L'activité métalinguistique est donc très réduite, et l'évolution des types d'emploi accompagne le mouvement argumentatif. En effet, si les auteurs commencent par concéder que le choix du terme est contestable (avec cependant la réserve exprimée par le modalisateur: "peut susciter un débat»), leur argument consiste ensuite à convoquer une observation pragmatique et, selon eux, incontestable de la « réalité » française, en laissant de côté les discours qui, selon eux toujours, ne lui correspondent pas :

Au-delà de ces positions idéologiques, la réalité du processus d'intégration suit son cours. Entre les représentations identitaires, les discours ou les revendications d'un côté, et la réalité des modes de vie, de consommation, des pratiques religieuses, des intermariages, des pratiques linguistiques, etc., on constate un écart parfois considérable. Souhaitée ou non, l'intégration, puis l'acculturation et enfin 
l'assimilation, des migrants, et plus encore celle de leurs enfants, s'est toujours réalisée en France, de façon plus ou moins rapide et plus ou moins facile (Vicher et al. $2011: 8)$.

Ces assertions, qui homogénéisent les publics et englobent toutes les situations, se placent à un très haut degré de généralité et d'abstraction, ce qui complique la contestation du propos - d'autant que la signification du terme n'a à aucun moment été explicitée. En outre, l'emploi d'Intégration en usage standard et en position de sujet syntaxique permet non seulement de ne désigner aucun acteur du processus, mais encore de renforcer le sémantisme du syntagme adjectival "souhaitée ou non ", en présentant l'intégration comme inéluctable puisqu'elle n'est le fait d'aucune volonté agissante identifiée.

La disjonction annoncée entre "discours » et " réalité » permet donc aux auteurs de prétendre résoudre le conflit en validant l'emploi et la prescription d'Intégration sans en expliciter le sens. En affirmant qu' Intégration est le terme convenable parce que l'intégration a lieu, les auteurs prescrivent une correspondance entre les propriétés du référent - les processus complexes qui suivent la migration durable d'une ou plusieurs personnes d'un pays ${ }^{9}$ vers un autre - et les critères d'appartenance (Kleiber 2001) à la classe d'occurrences délimitée par le concept d'intégration, sans pourtant avoir cherché à définir ou délimiter ni les unes ni les autres. Ces procédés permettent de nier la pertinence de la querelle de mots et donc de l'invisibiliser, contrairement à ce que laissait entendre l'énoncé ouvrant la section.

\subsection{Intégration face aux autres termes du paradigme}

En plus d'Intégration, trois des termes concurrents du paradigme sont actualisés dans la section analysée ici : Insertion, Acculturation et Assimilation. Il s'agit ici de voir comment les auteurs justifient le choix de l'un plutôt que des autres.

Dans l'avant-dernier paragraphe, on repère le mouvement de balancier entre emplois standard et autonymique décrit plus haut (3.2), cette fois autour de la dénomination Insertion : il est question de « dispositifs d'insertion sociale et professionnelle », puis du « terme d'insertion » et enfin des " actions et dispositifs d'insertion » (p. 9). Si d'Insertion, il est dit que le terme "rend donc lui aussi bien compte de la réalité du terrain professionnel et du champ éducatif concerné par ce référentiel», les auteurs ne justifient pas son rejet lorsqu'il s'est agi de nommer le référentiel. Pour leur part, Acculturation et Assimilation figurent en emploi standard dans la citation mise en exergue plus haut (3.2) et font figure de prolongements de l'intégration : leur validité n'est donc pas discutée, mais leur éviction n'est pas justifiée non plus.

Une dernière glose figure, autour de la dénomination Immersion: cette dernière est éliminée car elle masquerait la différence entre la situation des migrants et celle des étudiants et des expatriés, qui «n'ont absolument pas le même projet migratoire» (p. 9). Cette affirmation n'est cependant pas justifiée, bien que les situations puissent être plus poreuses que ce que les catégories employées laissent entendre.

A partir de tout ce qui précède, on peut faire l'analyse que les auteurs du référentiel jouent d'une dynamique entre effet de naturel et commentaire de la dénomination choisie, dans la mesure où il s'agit d'appuyer l'idée que dénomination et acte de nomination vont ici de soi. Si les auteurs sentent la nécessité de justifier leur choix pour anticiper des critiques potentielles, ils masquent, par l'ensemble des procédés mis 
au jour ci-dessus, le fait que leur choix nominatif exprime un rapport au réel, correspondant à un positionnement et à une deixis propre. Tout en définissant une prescription stricte, leur argumentaire repose sur l'idée qu'il ne s'agit pas d'un choix, puisqu'Intégration est la seule dénomination qui puisse convenir.

\section{Intégration : une nomination floue?}

Dans le contexte de la querelle de mots décrite plus haut (1), on aurait pu s'attendre à ce qu'un référentiel intitulé "Français Langue d'Intégration" inclue une définition générale explicite de la dénomination retenue, tant pour clarifier le propos que pour mieux le défendre. En effet,

En cas de conflit dénominatif, la première tâche à effectuer si l'on désire le résorber consiste à tenter d'en localiser la source - nous ne sommes pas d'accord pour dire "socialiste », ou "fasciste », tel ou tel régime, est-ce parce que nous n'attribuons pas le même sens aux mots "fasciste" et "socialiste", ou parce que nous divergeons dans notre appréciation du régime en question? (Kerbrat-Orecchioni $2000: 105-106)$

L'absence de définition explicite m'a amenée à adopter une méthodologie relevant de l'analyse du discours à entrée lexicale (Née et Veniard 2012), et ce dans une perspective contrastive. J'ai donc relevé toutes les occurrences des termes du paradigme désignationnel actualisés dans le référentiel (en excluant les occurrences d'Intégration apparaissant dans le contexte de la nomination Français Langue d'Intégration), afin de dégager, à travers l'étude des constructions syntagmatiques, les relations qui existent entre ces termes dans le cadre de ce discours socialement situé, et donc les programmes de sens qui y sont actualisés. Par ailleurs, si le référentiel n'inclut pas de définition à proprement parler des termes qui y sont employés, les trois sous-sections intitulées respectivement "L'intégration sociale ", "L'intégration économique » et «L'intégration citoyenne » (p. 11-12) retiendront mon attention ici.

\subsection{Intégration, Insertion, Acculturation et Assimilation dans le référentiel FLI}

Si les quatre dénominations figurent effectivement dans le référentiel, c'est dans des proportions très inégales : Intégration et Insertion y apparaissent respectivement 32 et 33 fois, tandis que Acculturation et Assimilation ne s'y trouvent que deux fois.

Huit adjectifs épithètes associés à Intégration et à Insertion entretiennent avec ces dénominations un rapport de détermination en restreignant leur contenu. Si plusieurs adjectifs leur sont communs, entretenant en première approche le brouillage, une observation plus fine des fréquences d'emploi marque des différences. On a ainsi :

\begin{tabular}{|l|c|c|}
\hline & INTÉGRATION (32) & INSERTION (33) \\
\hline \hline sans adjectif & 19 & 10 \\
\hline professionnelle & 1 & 13 \\
\hline économique & 5 & 2 \\
\hline
\end{tabular}




\begin{tabular}{|l|c|c|}
\hline socioprofessionnelle & 0 & 1 \\
\hline \hline sociale & 9 & 4 \\
\hline citoyenne & 6 & 1 \\
\hline sociolangagière & 3 & 0 \\
\hline culturelle & 0 & 1 \\
\hline socioculturelle & 0 & 1 \\
\hline
\end{tabular}

Tableau 1 : épithètes entretenant un rapport de détermination avec Intégration et Insertion. Une même occurrence pouvant être déterminée par plusieurs adjectifs, le total des adjectifs dépasse celui des occurrences. Les associations uniques constituent des hapax.

Si plus de la moitié des occurrences d'Intégration ne sont pas spécifiées, laissant ainsi la possibilité d'une interprétation large de la dénomination, les deux tiers des occurrences d'Insertion voient leur sens restreint à un domaine spécifique. La moitié des occurrences d'Insertion ont ainsi trait au champ professionnel ou économique, tandis que quatre d'entre elles sont associées au domaine social. Par contraste, quand Intégration est spécifié, c'est d'abord au champ social qu'il est fait référence, puis au domaine économique à égalité avec celui de la citoyenneté. Ce dernier élément marque d'ailleurs la spécificité d'Intégration par rapport à Insertion, dont l'association avec citoyenne constitue un hapax. Enfin, dans la mesure où pour les auteurs, l'apprentissage $\mathrm{du}$ français constitue «le moyen et la fin d'une intégration sociale, économique et citoyenne» (p. 7), on peut mettre en équivalence la dénomination Intégration sociolangagière avec celle d'Intégration en général.

On peut par ailleurs observer les syntagmes prépositionnels compléments du nom, qui fournissent des arguments à l'intérieur du SN. En particulier, le relevé des SP introduits par la préposition "par» permet de constater que si de nombreux moyens - non limités au domaine professionnel - par lesquels l'insertion peut avoir lieu sont nommés et identifiés, les facteurs de l'intégration identifiés dans le référentiel sont à la fois moins nombreux et exprimés d'une manière plus vague :

\begin{tabular}{|l|l|}
\hline intégration & insertion \\
\hline
\end{tabular}




\begin{tabular}{|l|l|}
\hline (faciliter l'int.) par l'apprentissage de la langue française & $\begin{array}{l}\text { ins. par le travail } \\
\text { (l'int. des migrants passe également) par leurs enfants } \\
\text { (l'int. passe donc) par la connaissance et le respect de ses } \\
\text { principes fondamentaux }\end{array}$ \\
interpersonnelles relations \\
ins. par les échanges du \\
quotidien \\
ins.par l'activité économique \\
ins.par l'emploi \\
ins.par le logement \\
ins.par le transport
\end{tabular}

Tableau 2: SP introduits par «par» compléments des noms Intégration et Insertion

De la même façon, Insertion est associé à de nombreux acteurs nommés comme tels (professionnels / acteurs / partenaires de l'Ins., structures d'Ins. + formateurs, bénévoles, coordinateurs des structures de formation linguistique) tandis qu'Intégration n'est associé qu'aux seuls acteurs de la formation linguistique.

Ces trois faisceaux d'éléments convergent pour, en première approche, faire de l'insertion une notion à la concrétude plus grande que celle d'intégration. Pour leur part, Acculturation et Assimilation sont présentés comme des horizons atteignables sur le chemin desquels l'intégration n'est qu'une étape : « l'intégration, puis l'acculturation et enfin l'assimilation, des migrants, et plus encore celle de leurs enfants, s'est toujours réalisée en France » (p. 8).

Pour finir, on examinera brièvement les nominations Intégration sociale, Intégration économique et Intégration citoyenne afin d'en faire ressortir les principaux éléments de sens actualisés dans le cadre du référentiel.

\subsection{Une intégration en trois volets : intégration sociale, économique et citoyenne}

Comme on l'a annoncé, chacune de ces trois nominations constitue le titre d'une soussection; l'ensemble est regroupé sous un titre associant les trois adjectifs: "Le français, langue d'intégration sociale, économique et citoyenne » (p. 11). Là encore, aucune définition explicite n'apparaît : c'est en examinant les contenus proposés qu'on peut mettre en évidence ce qui est nommé par les auteurs.

De façon assez attendue, Intégration sociale est associé aux « aspects pratiques de la vie quotidienne» (p. 11): relations interpersonnelles, transactionnelles, avec les administrations et les institutions éducatives. Si aucun horizon précis n'est posé comme balise, c'est «l'autonomie sociolangagière des migrants » (p. 11) qui est visée. Cette notion n'étant elle-même pas définie, les auteurs renvoient aux points de repère les différents niveaux du CECR.

La dénomination Intégration économique est quant à elle immédiatement remplacée par celle d'Insertion professionnelle, présentée comme une condition quasi-nécessaire de l'intégration (p. 12). Le but de l'insertion professionnelle est, de manière assez attendue là encore, l'entrée en formation professionnelle ou l'embauche. 
Enfin, le troisième volet de la nomination Intégration telle qu'actualisée dans le référentiel est aussi le plus saillant. On l'a dit en effet: si une spécificité de la dénomination Insertion est d'y être fréquemment restreinte au domaine professionnel, seule Intégration est parfois associé au champ de la citoyenneté. C'est d'ailleurs ce qui fonde en définitive, pour les auteurs, la différence entre les deux termes: pour eux, "l'insertion dans la société d'accueil par le travail, les relations interpersonnelles et les échanges du quotidien ne suffisent pas à qualifier l'intégration", et ce parce que " celle-ci suppose de surcroît une adhésion aux valeurs partagées par la communauté " ${ }^{10}$ (p. 12). L'horizon fixé est donc l'adhésion, et non pas seulement la connaissance ou le respect formel. L'intégration impliquerait donc non seulement une adaptation du comportement, mais aussi une transformation subjective profonde requérant l'adoption de nouvelles valeurs et l'abandon des valeurs non conformes. Le développement qui suit laisse bien peu de place à la contestation politique: les "principes démocratiques », qui sont revendiqués comme «le résultat d'une tradition historique, de luttes et de combats émancipateurs ", sont présentés comme un héritage figé et non négociable, des principes «intangibles et incontestables» (p. 12). Les " citoyens », qui « sont tenus de respecter [les lois] en toutes circonstances", n'ont pas leur mot à dire sur ces principes puisque " la démocratie établit une juste proportion entre la reconnaissance des droits imprescriptibles, la liberté des opinions et les devoirs des citoyens » (p. 12). Le référentiel pose donc le cadre d'un héritage verrouillé et incontestablement positif, qu'il est apparemment nécessaire de défendre auprès des nouveaux arrivants.

En ne définissant pas explicitement les termes qu'ils emploient, les auteurs du référentiel entretiennent un flou définitoire susceptible de rendre la contestation délicate. Ce flou est également décelable, à l'analyse, dans la moindre précision qui caractérise la nomination Intégration. L'analyse permet pourtant de mettre au jour des orientations idéologiques tangibles. Le praxème Intégration voit ainsi son programme de sens se réaliser à travers trois volets qui semblent en couvrir l'intégralité - laissant ainsi de côté une dimension plus explicitement culturelle. D'autre part, si Intégration sociale et Intégration économique sont actualisés à travers des aspects pragmatiques (autonomie au quotidien, emploi), Intégration citoyenne est le seul aspect qui fait intervenir une dimension subjective; dans le cadre de la formation linguistique, c'est cet aspect qui marque la spécificité du FLI puisque la notion d'intégration sociale est rabattue sur les niveaux du CECR, et celle d'intégration économique sur le FLP ${ }^{11}$. Enfin, si l'assimilation est présentée comme l'aboutissement atteignable de l'intégration, le très faible nombre d'occurrences ne permet pas la mise en contraste des deux termes, qui font pourtant l'objet d'un conflit de nomination récurrent.

\section{Conclusion}

La publication du référentiel FLI constitue un acte de re-nomination appuyé sur un agencement de pouvoir qui renforce la portée de l'argumentation déployée par les auteurs : placés en position d'expertise par l'institution, leur parole voit son autorité renforcée. Par ailleurs, si pour Monte et Oger (2015), l'autorité des discours institutionnels a ceci de spécifique qu'elle ne se construit pas par la force mais par l'effacement du dissensus, on doit relever qu'ici, cette autorité repose malgré tout sur 
un arsenal juridique qui permet d'imposer une nomination ainsi que ses effets dans la réalité extra-linguistique, et ce malgré les contestations qui ont eu lieu.

Toute nomination relève par ailleurs de phénomènes de coréférence : chaque objet du monde est dénommé par approximations successives qui s'ajoutent les unes aux autres pour approcher l'objet (Chanay 2001). Cependant, les dénominations auxquelles on a affaire ici font l'objet d'un conflit portant tant sur la façon de décrire et d'analyser le processus social en œuvre que sur ce qui est souhaité. On a mis en évidence la manière dont les auteurs cherchent à masquer leur positionnement, en s'effaçant énonciativement ainsi qu'en adoptant une posture surplombante qui leur permet de neutraliser toute objection. S'ils se présentent comme conscients de la querelle de mots dans laquelle ils interviennent, la résolution qu'ils en proposent repose sur la présentation partiale de deux positions adverses, puis leur éviction en faveur d'un appel au pragmatisme. En prétendant qu'il n'y a pas d'alternative nominative alors qu'ils effectuent un choix, qui plus est lié à une prise de position axiologique, les auteurs nient la pertinence du dissensus tout en s'y positionnant, ce qui ne permet pas de résoudre le conflit de nomination. Là où Micheli (2013) identifie deux types d'enchaînements argumentatifs prototypiques permettant de justifier l'usage ou le non-usage d'un mot, le premier reposant sur l'applicabilité référentielle du terme, le second sur sa charge dialogique et ses effets sur l'allocutaire, on a ici affaire à une configuration différente dans la mesure où il n'y a ni glose définitoire, ni mention d'une quelconque charge dialogique : la validité de la nomination est défendue par un recours à l'évidence.

Par ailleurs, les auteurs ne prennent en charge ni l'explicitation de la signification que revêtent pour eux les dénominations actualisées, ni la définition de l'horizon sociétal qu'ils souhaitent voir advenir: ce double brouillage constitue un procédé discursif efficace qui rend difficile l'appréhension des catégories par le lecteur. Une analyse plus fine met cependant en évidence des éléments du programme de sens d'Intégration que les auteurs actualisent et construisent à la fois, et qui correspondent à des orientations idéologiques spécifiques alors imposées par l'institution.

L'ensemble de ces analyses permet d'apporter un éclairage critique supplémentaire sur le référentiel FLI, ses conditions de production et ses contenus, dans la continuité des débats intervenus depuis sa sortie.

\section{BIBLIOGRAPHIE}

\section{Corpus}

Vicher, Anne, Hervé Adami, Amandine Bergère [et al.]. 2011. Référentiel FLI - Français Langue d'Intégration. Direction de l'Accueil, de l'Intégration et de la Citoyenneté. En ligne : http:// www.immigration.interieur.gouv.fr/content/download/38544/292981/file/FLI-Referentiel.pdf, (Consulté le 14 juillet 2015)

Références 
Amossy, Ruth. 2006 [2000]. L'argumentation dans le discours (Paris : Colin).

Bonnafous, Simone. 1992. «Le terme intégration dans le journal Le Monde : sens et non-sens », Hommes et Migrations 1154, 24-30

Bourdieu, Pierre. 1993. « Esprits d'État. Genèse et structure du champ bureaucratique », Actes de la recherche en sciences sociales 96/97, 49-62

Bruneau, Aurélie, Véronique Castellotti, Marc Debono, Cécile Goï \& Emmanuelle Huver. 2012. «Langue(s) et insertion : quelles relations, quelles orientations ?», Diversité 170, 185-192

Castellotti, Véronique. 2008. «Introduction. Vers la construction d'une école et d'une société plurielles : des notions en débat, des orientations à construire », Glottopol 11, 2-12

Chanay, Hugues Constantin de. 2001. "La dénomination : perspective discursive et interactive », Cahiers de Praxématique 36, 169-188

Dubar, Claude. 1999. « Réflexions sociologiques sur la notion d'insertion ». Charlot, Bernard \& Dominique Glasman (éds), Les jeunes, l'insertion, l'emploi (Paris : Presses universitaires de France), 29-37

Étienne, Sophie. 2004. « Nouvelles orientations gouvernementales et bouleversements dans le monde de la formation ", Études de linguistique appliquée 133/1, 109-117

Fédération des centres sociaux et socioculturels de Paris. 2012. «Le Français Langue d'Intégration (FLI) : un concept et un référentiel qui heurtent les valeurs et les principes des centres sociaux associatifs parisiens ». En ligne $:$ http://paris.centres-sociaux.fr/files/2012/04/Microsoft-WordDeclaration_FLI.pdf] (Consulté le 22 janvier 2016)

«Français langue d'intégration (FLI) : réponse des acteurs de terrain, des scientifiques et des universitaires qui y sont descendus ». En ligne : https://blogs.mediapart.fr/edition/les-invitesde-mediapart/article/161111/francais-langue-dintegration-fli-reponse-des-ac (Consulté le 9 janvier 2016)

« Flic ? Le français, langue d'une intégration contrôlée ». En ligne : https://blogs.mediapart.fr/ edition/les-invites-de-mediapart/article/091111/flic-le-francais-langue-dune-integration-contro (Consulté le 17 janvier 2016)

Gaspard, Françoise. 1992. « Assimilation, insertion, intégration : les mots pour "devenir Français" ", Hommes et migrations, 1154, 1423

Grange, Juliette. 2005. «Que veut dire intégration? Histoire d'une notion », Ferry, Vincent, PieroDominique Galloro \& Gérard Noiriel (éds). 20 ans de discours sur l'intégration (Paris : L'Harmattan), 41-47

Huver, Emmanuelle, Didier de Robillard, Aurélie Bruneau [et al.]. 2011. « Le FLI peut-être, mais... ». En ligne : http://dynadiv.univ-tours.fr/medias/fichier/manifeste_1319447605683.pdf? INLINE=FALSE (Consulté le 22 janvier 2016)

Kerbrat-Orecchioni, Catherine. 2000. «L'analyse des interactions verbales. La notion de "négociation conversationnelle" : défense et illustration », Lalies 20, 63-141

Kleiber, Georges. 1997. « Sens, référence et existence : que faire de l'extra-linguistique? », Langages 127, 9-37

Kleiber, Georges. 2001. « Remarques sur la dénomination », Cahiers de praxématique 36, 21-41 
Laacher, Smaïn \& Abdelmalek Sayad. 1999. «Insertion, intégration, immigration : la définition des mots est toujours un enjeu dans les luttes sociales », Charlot, Bernard \& Dominique Glasman (éds.), Les jeunes, l'insertion, l'emploi (Paris : Presses universitaires de France), 166-185

Lapeyronnie, Didier. 2003. «Quelle intégration?», Loche, Bernard \& Christophe Martin (éds.). L'insécurité dans la ville : changer de regard (Paris : L'Æiil d'Or), 95-98

Lebreton, Emilie. 2014. «Les formations linguistiques au prisme de la logique de marché. Quelles conséquences sur le «terrain"? », Savoirs et formation - Recherches et pratiques, 4, 92-103

Leclercq, Véronique. 2012. «La formation des migrants en France depuis l'alphabétisation des années 60 », Adami, Hervé \& Véronique Leclercq (éds.). Les migrants face aux langues des pays d'accueil (Villeneuve d'Ascq : Presses Universitaires du Septentrion), 173-196

Lemière, Jacques. 2006. « De l'importance et de l'urgence d'étendre dans le travail social, la critique des catégories d'intégration et d'immigré », Prieur, Elisabeth, Emmanuel Jovelin \& Martine Blanc (éds.). Travail social et immigration (Paris : L'Harmattan), 91-103

Lochak, Danièle. 2006. «L'intégration comme injonction. Enjeux idéologiques et politiques liés à l'immigration », Cultures \& Conflits 64, 131-147

Logez, Guillaume. 2005. « L'intégration sous les discriminations. Une action publique qui ne va pas de soi ». Ferry, Vincent, Piero-Dominique Galloro \& Gérard Noiriel (éds.). 20 ans de discours sur l'intégration (Paris : L'Harmattan), 197-201

Micheli, Raphaël. 2012. «Les visées de l'argumentation et leurs corrélats langagiers : une approche discursive ». Argumentation et Analyse du Discours 9. En ligne : http://aad.revues.org/ 1406 (Consulté le 11 novembre 2013)

Micheli, Raphaël. 2013. « Les querelles de mots dans le discours politique : modèle d'analyse et étude de cas à partir d'une polémique sur le mot "rigueur" ", Argumentation et Analyse du Discours 10. En ligne : https://aad.revues.org/1446 (Consulté le 6 novembre 2013)

Moirand, Sophie. 2003. « De la nomination au dialogisme : quelques questionnements autour de l'objet de discours et de la mémoire des mots ", Cassanas, Armelle, Aude Demange, Bénédicte Laurent \& Aude Lecler (éds), Dialogisme et nomination (Montpellier : Publications Montpellier 3), 27-61

Monte, Michèle \& Claire Oger. 2015. « La construction de l'autorité en contexte. L'effacement du dissensus dans les discours institutionnels », Mots. Les langages du politique 107, 5-18

Née, Émilie \& Marie Veniard. 2012. « Analyse du Discours à Entrée Lexicale (A.D.E.L.) : le renouveau par la sémantique?", Langage et société 140, 15-28

Rabatel, Alain. 2003. «L'effacement énonciatif dans les discours représentés et ses effets pragmatiques de sous - et de sur-énonciation », Estudios de Lengua y Literatura Francesas 1 (14), 33-61

Siblot, Paul. 2001a. « De la dénomination à la nomination. Les dynamiques de la signifiance nominale et le propre du nom », Cahiers de praxématique 36, 189-214

Siblot, Paul. 2001b. « Nomination », Détrie, Catherine, Paul Siblot \& Bertrand Vérine (éds). 2001. Termes et concepts pour l'analyse du discours : une approche praxématique (Paris : Champion), 205-207

Siblot, Paul. 2007. « Nomination et point de vue : la composante déictique des catégorisations lexicales ", Cislaru, Georgeta [et al.] (éds.) L'acte de nommer. Une dynamique entre langue et discours (Paris : Presses de la Sorbonne Nouvelle), 25-38 
Vadot, Maude. 2011. « Le Contrat d'Accueil et d'Intégration : implications des contraintes institutionnelles sur les pratiques de formation linguistique ", Travaux de didactique du FLE 65-66, 243-261

Vadot, Maude. 2014 « Les conditions de travail des formateurs et formatrices dans le contexte actuel de la formation de base ", Savoirs et formation - Recherches et pratiques 4, 104-124

Vadot, Maude (à paraître en 2016). « Qu'est-ce que l'intégration? Analyse des discours de formateurs et formatrices intervenant auprès de migrants adultes en France ", Actes $d u$ Symposium Intégration Linguistique des Migrants Adultes, Conseil de l'Europe, 29 mars - 1er avril 2016 (Berlin : Mouton De Gruyter)

Wieviorka, Michel. 2001. «Faut-il en finir avec la notion d'intégration? » Les Cahiers de la Sécurité Intérieure 45, 9-20

\section{NOTES}

1. Que de Chanay désigne, dans le même volume, par « acte de dénomination » (Chanay $2001: 2$ ). 2. Michel Aubouin (alors directeur de la Direction de l'Accueil, de l'Intégration et de la Citoyenneté) et Xavier North (alors délégué général à la langue française et aux langues de France - DGLFLF)

3. En l'occurrence, des formateurs intervenant auprès de publics migrants en France en 2013.

4. Le référentiel comportant à proprement parler 36 pages, je ne pourrai en citer ici que de courts extraits. Le texte complet est consultable sur le site du Ministère de l'Intérieur (cf. bibliographie).

5. Selon le décret $n^{\circ}$ 2011-1266, le label visait à «identifier les organismes habilités à délivrer une formation linguistique ». Mais les appels d'offres publiés par l'OFI depuis n'ont jamais fait mention de l'exigence du label, même si détenir ce dernier a pu favoriser l'obtention du marché.

6. trois cent trente-six organismes de formation étaient labellisés en août 2015 (http:// www.immigration.interieur.gouv.fr/content/download/40160/309288/file/DISPOSITIF-FLI-TB-5aout.xls, consulté le 17 janvier 2016).

7. Notamment dans les universités de Lorraine, Strasbourg, Pau, Cergy Pontoise et Caen. Paris Ouest Nanterre avait ouvert master et DU FLI mais les a fermés à la rentrée 2015.

8. Le " prédicat de réalité » de Siblot (2001b : 207) rejoint ainsi la " présupposition existentielle » de Kleiber (1997 : 18).

9. Dans le référentiel, c'est la situation qui est envisagée.

10. En complément du référentiel, un livret élaboré par la DAIC liste ce qui est désigné comme les 25 valeurs de la République Française (http://crapt-carrli.gip-fcip-alsace.fr/images/fli/ fli_2014/enseigner_les_valeurs.pdf, consulté le 14 juillet 2015). A destination des formateurs, ce livret assure donc le transfert opérationnel de la notion d'intégration citoyenne vers les formations linguistiques.

11. Français Langue Professionnelle. 


\section{RÉSUMÉS}

Le présent article vise à étudier l'articulation entre argumentation et nomination à partir du conflit nominatif réactivé par la parution du Référentiel Français Langue d'Intégration (FLI), dans une perspective d'analyse de discours praxématique et sociolinguistique. Après avoir resitué brièvement la querelle qui touche la nomination Intégration, nos analyses poursuivront un double objectif. Il s'agira d'une part de décrire une situation dans laquelle le mot devient un enjeu de l'argumentation : par quelles stratégies socio-discursives les locuteurs légitiment-ils et justifientils leur choix ? On cherchera d'autre part à documenter et à éclairer le conflit en mettant au jour, dans une démarche sémasiologique inspirée de l'analyse de discours à entrée lexicale, des éléments du programme de sens du praxème Intégration que les auteurs, de façon dialectique, actualisent et contribuent en même temps à imposer dans le champ de la formation linguistique. L'ensemble du propos constitue un éclairage critique supplémentaire sur le référentiel FLI, ses conditions de production et ses contenus, dans la continuité des débats intervenus depuis sa sortie.

The article aims at describing the articulation between argumentation and nomination based on the nominative conflict revived by the publication of the Référentiel Français Langue d'Intégration (FLI), in the viewpoint of a praxematic and sociolinguistic discourse analysis. After having briefly presented the debates which occur about the nomination Integration, my analyses follow two complementary goals. I first describe a situation in which the word becomes the object of the argumentation. By which sociodiscursive strategies speakers legitime and justify their nominative choice ? By a discourse analysis based on a lexical entry, I then try to document and enlighten the conflict by disclosing some elements of the meaning program of the praxem Integration that the authors, in a dialectic way, actualize and contribute to impose in the meantime. The whole text constitutes an additional and critical lighting about the FLI frame of reference, its production conditions and its contents, in the continuity of the debates which occurred since it was released.

\section{INDEX}

Mots-clés : analyse de discours à entrée lexicale (ADEL), argumentation, Français Langue d'Intégration (FLI), nomination polémique, programme de sens

Keywords : argumentation, controversial nomination, discourse analysis based on a lexical entry, Français Langue d'Intégration (FLI), meaning program

\section{AUTEUR}

\section{MAUDE VADOT}

Université Paul Valéry Montpellier 3, ITIC, Dipralang EA739 